\title{
Applications of Heuristics and Metaheuristics in Power Systems
}

\author{
Ruben Romero, ${ }^{1}$ Edgar M. C. Franco, ${ }^{2}$ and Massoud Rashidinejad ${ }^{3}$ \\ ${ }^{1}$ University of the State of São Paulo (UNESP), Ilha Solteira, SP, Brazil \\ ${ }^{2}$ State University of Western Paraná (UNIOESTE), Foz do Iguazu, PR, Brazil \\ ${ }^{3}$ Shahid Bahonar University of Kerman 76169, Kerman, Iran \\ Correspondence should be addressed to Ruben Romero, ruben@dee.feis.unesp.br \\ Received 11 March 2012; Accepted 11 March 2012
}

Copyright ( 2012 Ruben Romero et al. This is an open access article distributed under the Creative Commons Attribution License, which permits unrestricted use, distribution, and reproduction in any medium, provided the original work is properly cited.

The electricity industry has undergone profound changes in recent years, resulting in significant changes in economic relations in the generation, transmission, and distribution sectors. This is also leading to important changes in operations management and planning processes relating to different sectors of generation, transmission, distribution, and commercialization of electricity.

In this context, the optimization techniques for application in electrical system operation and planning are becoming increasingly important, especially with the growth in size and complexity of new mathematical models related to optimization problems of electric power systems.

In the optimization of operation and planning problems of electric power systems, optimization techniques used are from the field of operations research. These techniques can be classified into two major groups: (1) exact methods and (2) approximate methods.

Exact methods can be analytical or classical optimization techniques. Analytical exact methods have virtually no application in large and complex problems, and classical optimization techniques include the use of optimization techniques such as linear programming, nonlinear programming, mixed integer linear, and mixed integer nonlinear.

Approximate methods include heuristics and metaheuristics. It should be noted that the larger and more complex the mathematical model of a problem, the more efficient the metaheuristics when compared with other optimization techniques. This special issue presents various optimization proposals related to the operation and planning of power systems using heuristics and metaheuristics.

One paper addresses the problem of multicriteria reconfiguration of distribution network with distributed generation according to the criterion of minimum power loss under normal conditions and the criterion of power supply reliability under postemergency conditions. In this case an specialized ant colony algorithm is used to solve the problem for minimum loss reconfiguration of distribution network. Additionally, some interesting results are presented with data from the Central Power System of Mongolia.

Another paper presents a novel mathematical model for transmission network expansion planning. The main contribution is to consider phase shifter as new elements of the transmission system expansion together with other traditional components such as transmission lines and conventional transformers. There are few proposals of this kind in the specialized literature. It should be noted that the results of this kind of expansion planning require lower investment since the redistribution of the power flow as a result of the phase shifter allows for better use of the transmission lines capacity.

One of the papers assesses the reliability of distribution systems using artificial intelligence methods. The paper presents an artificial neural networks (ANNs) version for evaluating the reliability of distribution power systems. In this case, ANN is used to predict the reliability of the power system using historical data, and the method is constructed according to the back-propagation learning rule. At the same time, the system average interruption frequency index (SAIFI), and the system average interruption duration index (SAIDI) of the real distribution system are computed and compared with results generated by a network method. The results presented are promising.

An evolutionary optimization of electric power distribution planning by means of the dandelion code has been manifested in one of the papers. In this paper, the problem is solved using a genetic algorithm that, with the help 
of a coding based on the dandelion code permits solving larger instances of the problem. Using this optimization technique, the authors can solve large size problems such as, for example, electrical networks with 4000 buses and 20 substations.

One other paper describes a specialized genetic algorithm for solving the static and multistage transmission network expansion planning. In this case, an initial population is generated using fast and efficient heuristic algorithms, a better implementation of the local improvement phase is implemented, and an efficient solution of linear programming problems is proposed. Critical comparative analysis is made between the proposed genetic algorithm and traditional genetic algorithms. Results using some known systems show that the proposed specialized genetic algorithm presents higher efficiency in solving the static and multistage transmission network expansion planning.

The final paper on this issue presents two multiobjective optimization methods for congestion management in deregulated power systems. The paper proposes two effective methods for transmission congestion alleviation in deregulated power systems. Congestion or overload in transmission networks is alleviated by rescheduling of generators and/or load shedding. The two objectives that are optimized in this paper, transmission line overload and congestion cost, are conflicting. Multiobjective fuzzy evolutionary programming and nondominated sorting genetic algorithm-II methods are used to solve this problem. The quality and usefulness of the algorithm are tested on the IEEE30 bus system.

Ruben Romero

Edgar M. C. Franco

Massoud Rashidinejad 

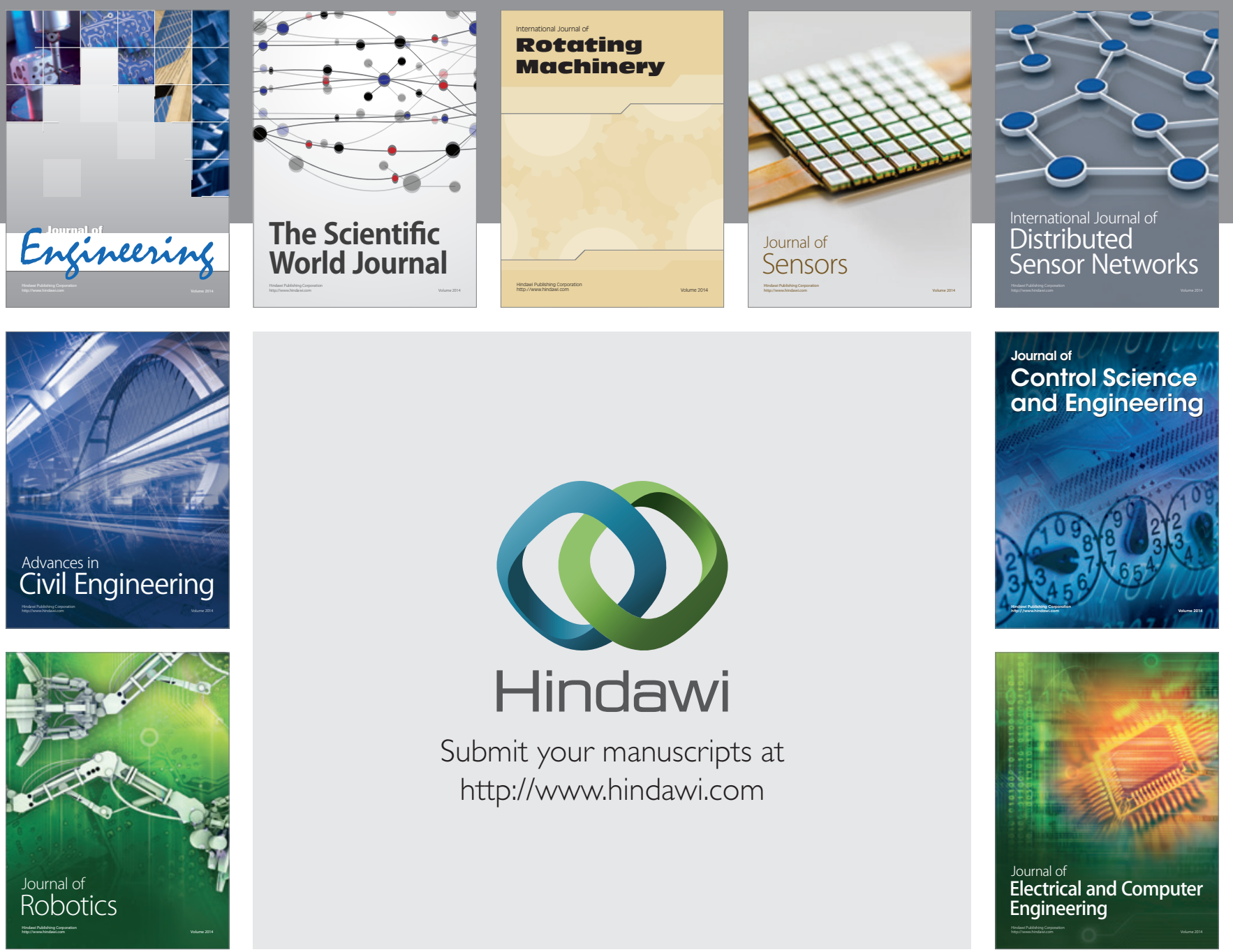

Submit your manuscripts at

http://www.hindawi.com
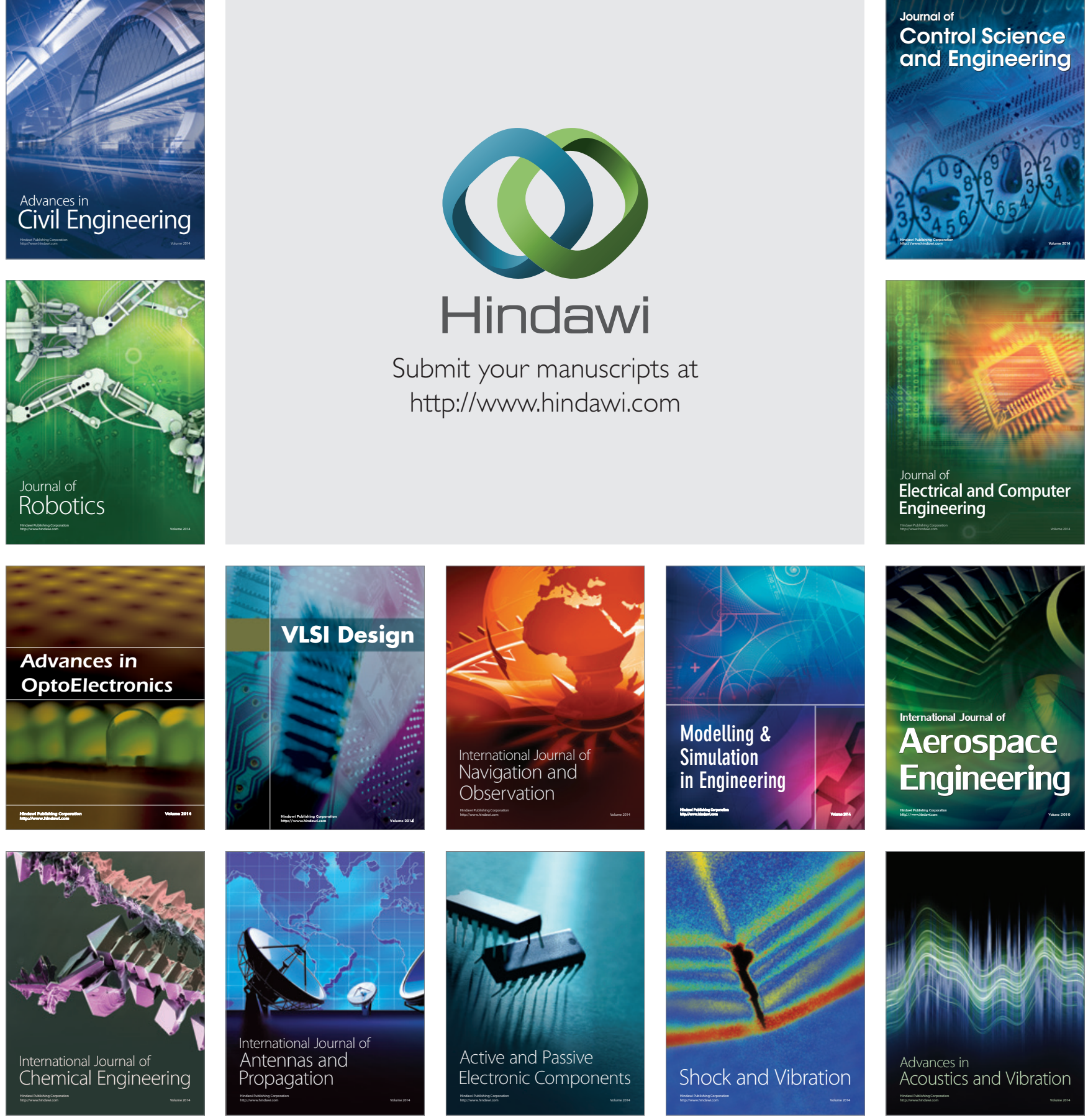